\title{
Erratum: Layer-dependent quantum cooperation of electron and hole states in the anomalous semimetal $\mathrm{WTe}_{2}$
}

\author{
Pranab Kumar Das, D. Di Sante, I. Vobornik, J. Fujii, T. Okuda, E. Bruyer, A. Gyenis, B.E. Feldman, J. Tao, R. Ciancio, \\ G. Rossi, M.N. Ali, S. Picozzi, A. Yadzani, G. Panaccione \& R.J. Cava
}

Nature Communications 7:10847 doi: 10.1038/ncomms10847 (2016); Published 29 Feb 2016; Updated 13 Apr 2016

This Article contains errors in the numbering of the references in the text.

The third sentence of the second paragraph should only refer to reference 2, not references 2 and 3 . The correct sentence is:

'It exhibits an extremely large uniaxial positive magnetoresistance with no saturation up to a magnetic field as high as $60 \mathrm{~T}$ (ref. 2 ), which has been attributed to perfect electron and hole compensation.'

All subsequent reference numbers in the text refer to the previous paper in the reference list. For example, 4 in the text refers to the third reference in the list, 5 refers to the fourth paper in the list and so on throughout up to and including 32 in the text, which refers to the 31 st paper in the reference list.

The 32nd reference in the list should be cited in the first sentence of the final paragraph of the Methods section. The correct sentence is:

'To calculate bulk Fermi surfaces reported in Fig. 3 (right panel), we adopt a two step procedure: (i) first, we projected the bulk Hamiltonian onto a basis made of $s$ and $d \mathrm{~W}$-centered, and $s$ and $p$ Te-centered orbitals, for a total of 112 Wannier functions, by means of the WANNIER90 package ${ }^{32}$; (ii) subsequently, the Wannier Hamiltonian is used to build up the bulk Green's function as $G(\mathbf{k}, w)=1 /(w-H(\mathbf{k})+i \delta)$, which, in turn, gives the Fermi surface maps at the chosen binding energy, $w$.'

This work is licensed under a Creative Commons Attribution 4.0 International License. The images or other third party material in this article are included in the article's Creative Commons license, unless indicated otherwise in the credit line; if the material is not included under the Creative Commons license, users will need to obtain permission from the license holder to reproduce the material. To view a copy of this license, visit http://creativecommons.org/licenses/by/4.0/ 